\title{
Laboratory-tutorial activities for teaching probability
}

\author{
Michael C. Wittmann,* Jeffrey T. Morgan, and Roger E. Feeley \\ Department of Physics and Astronomy, University of Maine, Orono, Maine 04469-5709, USA
}

(Received 21 February 2006; published 2 August 2006)

\begin{abstract}
We report on the development of students' ideas of probability and probability density in a University of Maine laboratory-based general education physics course called Intuitive Quantum Physics. Students in the course are generally math phobic with unfavorable expectations about the nature of physics and their ability to do it. We describe a set of activities used to teach concepts of probability and probability density. Rudimentary knowledge of mechanics is needed for one activity, but otherwise the material requires no additional preparation. Extensions of the activities include relating probability density to potential energy graphs for certain "touchstone" examples. Students have difficulties learning the target concepts, such as comparing the ratio of time in a region to total time in all regions. Instead, they often focus on edge effects, pattern match to previously studied situations, reason about necessary but incomplete macroscopic elements of the system, use the gambler's fallacy, and use expectations about ensemble results rather than expectation values to predict future events. We map the development of their thinking to provide examples of problems rather than evidence of a curriculum's success.
\end{abstract}

DOI: 10.1103/PhysRevSTPER.2.020104

PACS number(s): 01.40.Fk, 01.50.Qb, 02.50.Cw

\section{INTRODUCTION}

Though probability and simple statistics are common in our society (ranging from baseball batting averages to polling data with its statistical measures), we rarely teach the concepts in our introductory physics courses. The learning of the concept of probability has been studied in great detail in the area of mathematics and statistics-for example, in Refs. 1 and 2-and there is a thorough (though slightly outdated) bibliography on the topic. ${ }^{3}$ Likely because probability is rarely taught in introductory physics, it has not been studied much by physics education researchers. The little research that has been carried out on teaching probability has been related to developing ideas useful for teaching quantum physics, and the data are mostly from advanced physics students. ${ }^{4-6}$ Our study looks at a new population of physics students taking Intuitive Quantum Physics, a general education physics course at the University of Maine, developed by members of the Physics Education Research Laboratory (in alphabetical order: Katrina E. Black, David E. Clark, Roger E. Feeley, Jeffrey T. Morgan, Eleanor C. Sayre, and Michael C. Wittmann). We have created a set of activities for teaching probability in a way that can help non-science-major students later learn concepts of quantum physics. We describe the effect of our course on student understanding of probability and probability density in classical situations for a population that, anecdotally, has little mathematical confidence or science background.

A goal of our course has been to make physics concepts accessible to students who are on their way to learning the introductory ideas of quantum physics. We use the Copenhagen interpretation of the wave function in Intuitive Quantum Physics, and students are required to understand that $|\Psi|^{2}$ gives the local probability density of finding a particle at a given location. Thus, we emphasize the ratio of being found in a region compared to the likelihood of being found in all possible regions. To help students understand this concept, we begin by teaching about probability in nonphysics situations and then move into discussions of probability density in physical situations such as a ball toss or oscillating glider. Our work on probability density is built on ideas first introduced by Jolly et al. ${ }^{7}$ and Bao and Redish, ${ }^{4}$ but the activities begin with a far more basic definition of probability in familiar situations. Furthermore, we teach in a different instructional format to our population of nonscience majors using new teaching tools which extend previous tools. We describe the students' mathematical background and expectations about science knowledge below.

In this paper, we describe the curriculum so that others may adapt it to their own needs. We describe student reasoning as they learn from the curriculum so that we may help inform the choices of those adapting the materials. The teaching materials are accessible at Ref. 8, and we only summarize them in this paper. Research data are given based on the Fall, 2005, semester in which we were best able to track student learning on the topic of probability density. Results are consistent with results gathered from previous semesters in which we do not have as comprehensive a set of longitudinal data. We describe data from several ungraded pre- and post-tests and two examination questions, as well as informal observations which are consistent with our research data.

\section{STUDENT DEMOGRAPHICS AND COURSE DESIGN}

The University of Maine, a Carnegie research I institution, is the flagship campus of the Maine state university system. The Department of Physics and Astronomy offers a course in "Descriptive Physics" which is taken primarily by nonscience majors needing a laboratory science course for core-curriculum "general education" graduation requirements. The course format was originally designed by Harrington and Prather. ${ }^{9}$ The course is taught with three hours of laboratory time and three hours of lecture time. All lab time is placed between the first and second lectures in a given week. Class sizes in this course are typically between 40 and 
TABLE I. Course outline. Each unit has specific goals concerning content knowledge and the nature of science.

\begin{tabular}{|c|c|c|c|c|}
\hline & & \multicolumn{3}{|c|}{ Nature of science questions } \\
\hline & & How do you know? & How can you explain? & Why do you believe? \\
\hline \multirow[t]{3}{*}{ Content Unit } & $\begin{array}{l}\text { Unit 1: optics and wave physics } \\
\text { - Light travels outward in a straight line } \\
\text { from every source point } \\
\text { - Superposition of visible waves on a } \\
\text { spring and in water } \\
\text { - Water and light interference } \\
\text { - Wave-particle duality-the great dilemma! }\end{array}$ & "I saw it" & $\begin{array}{l}\text { "It's like something } \\
\text { in real life" }\end{array}$ & "I saw it" \\
\hline & $\begin{array}{l}\text { Unit 2: a new toolbox } \\
\text { - Energy diagrams } \\
\text { - Classical probability } \\
\text { - Curviness of graphical functions } \\
\text { - A graphical interpretation of the } \\
\text { Schrödinger equation }\end{array}$ & "You told me" & $\begin{array}{l}\text { "It's like something } \\
\text { else I know" }\end{array}$ & "I thought about it" \\
\hline & $\begin{array}{l}\text { Unit 3: topics in quantum physics } \\
\text { - Quantization in finite wells, bound states } \\
\text { - Spectroscopy } \\
\text { - Models of molecules } \\
\text { - Quantum tunneling }\end{array}$ & "I figured it out" & $\begin{array}{l}\text { "It's consistent with } \\
\text { these other things" }\end{array}$ & $\begin{array}{l}\text { "I'm not sure I do, } \\
\text { but I can think } \\
\text { about it, anyway" }\end{array}$ \\
\hline
\end{tabular}

80. In Fall, 2005, 47 students began the course and 43 completed it.

Students signing up to take the Descriptive Physics course typically have unfavorable expectations and attitudes about physics. Informal results show that students enter the course expecting (and worried about) the mathematical nature of the course. More formally, ${ }^{10}$ we find that they enter the course expecting to use "memorize and repeat" learning methods and do not have a view of physics that includes conceptual understanding. Based on several semesters of data using the Maryland Physics Expectations Survey 2 (MPEX 2), ${ }^{11,12}$ we find that students typically enter the course having roughly $50 \%$ unfavorable and $25 \%$ favorable expectations about conceptual learning. These scores are substantially lower than a typical introductory class, as measured by the original MPEX. ${ }^{12}$ The high number of neutral responses is consistent with our observations of other non-science-major courses at the University of Maine or high school courses.

We have developed the Intuitive Quantum Physics course to best match student concerns and abilities while teaching them a meaningful physics course on an interesting topic. Starting with optics and waves, students develop simple ideas of quantum physics while continually connecting ideas to their everyday life. We use as little algebra as possible and emphasize other valid methods of reasoning, such as graphical analysis and qualitative reasoning. Where possible, we have students build an understanding of physics from easily observable phenomena, giving students touchstone concepts when dealing with more complicated topics. For example, superposition concepts are always chained back to the observed superposition of waves on a spring; wave and particle interpretations of quantum particles are chained back to the two-slit interference situation in which individual particles arrive on a screen and eventually fill in an interference pattern; ramped potential energy diagrams are chained back to carts on ramps and square well potential energy diagrams are chained back to a cart bouncing back and forth "frictionlessly" and perfectly elastically between two hard walls. Most material is introduced in a single, three-hour-long labtutorial period. The lab-tutorial includes individual, small group, and large group (full class) activities.

The Intuitive Quantum Physics course is split into three units in which students develop skills prerequisite to understanding quantum physics, create a "toolbox" with which to study the quantum world, and discuss applications of quantum physics to the real world. Table I summarizes the course structure. We provide this information to situate the activities on probability within the larger context of the course. We teach probability to provide a language which lets students understand the behavior of particles in the wave-particle duality two slit experiment: where might the next particle arrive on screen? The move to a description of probability density arises naturally in the course, though students have few tools to work with. Unit II, "a new toolbox," is designed to introduce the most basic ideas of probability (as defined below) and probability density, along with energy and graphical representations of the second derivative of a function. The ideas taught are used to discuss probability density in bound-state problems and other quantum physics situations.

\section{TEACHING PROBABILITY}

To summarize the instructional materials for teaching probability, we introduce concepts of probability by first looking at discrete, nonphysics systems (such as coins and dice) and then study macroscopic physical systems (such as balls tossed in the air or oscillating air gliders). Later, students connect these ideas to potential energy graphs for the same physical situations - they must learn to connect poten- 
tial and kinetic energy and consider where the object is moving slowest and spends the most time.

As discussed in the next section, we find that students enter our courses unable to apply many of the ideas about probability. Many apply the gambler's fallacy to situations, while others are unclear about the interpretation of probability density and the link between an object's speed and the likelihood of finding it in a region of space.

The primary ideas we wish students to learn are the following.

- the sum of probabilities of outcomes to an event equals 1 (or $100 \%)$,

- for small $n$, expected distribution results can be predicted, but most likely do not match actual outcomes of ensembles of events,

- in physical systems, we can compare the time in one region of space to the total time in all regions of space as a way of finding the probability of being located in that region,

- relative time in a region of space is determined by the speed of the object in that region.

We visit these concepts twice, in lab-tutorial 5 (Probability) and 7 (Probability and Energy). In lab-tutorial 6, we introduce concepts of kinetic and potential energy, create potential energy diagrams for several "touchstone" systems (harmonic oscillators, ramps, square wells, and barriers), and do not discuss probability or probability density. The descriptions below come primarily from lab-tutorial 5 (Probability) unless noted.

\section{A. Discrete systems}

We introduce the idea of probability by asking students to consider a person reaching into a box containing ten balls (five are checkered, three are striped, and two are solid) and removing a ball. Students are asked to find the probability of picking each type of ball and finding the total probability. We thus establish the idea of a probability of 1 (or 100\% chance of an event occurring, in this case "a ball being picked"). Students typically have no problem with this idea.

Students follow up with a series of questions about coin tosses. After ten tosses, students expect five heads and five tails. Is six of one and four of the other a surprise? Is ten of one and zero of the other a surprise? Students carry out ten coin tosses individually and then work in groups to compare answers. A range of results helps them discuss variability of results.

Students are next asked to toss three coins at once. Results will be variations of HHH (three heads, no tails), HHT, HTT, and TTT. Coin order matters, and students are asked to predict all possible outcomes and discuss the probability of finding each. At this point, we introduce a histogram representation, shown in Fig. 1 with possible data from an experiment in which coins were tossed 23 times.

Once students have predicted results, they are asked to carry out the experiment and toss three coins in unison a total of eight times. Students compare a histogram of their actual data to the prediction. Results vary from student to student. Again, there is an opportunity to discuss the difference between predictions and results. Students also discuss the role

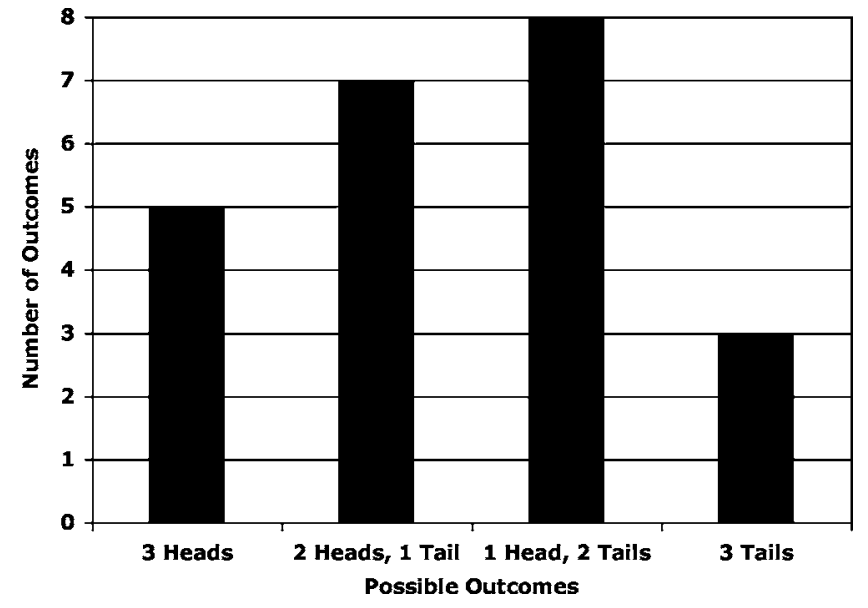

FIG. 1. Representative probability histogram chart for the three coin toss.

of larger ensembles of results after combining all results from a table.

Students move on to consider distinguishable dice (two differently sized or colored dice are used in class). They roll the dice together, keeping track of each individual die's result and the sum of the two. Again, a probability histogram is created and its results are compared to the 36 possible combinations of dice tosses.

\section{B. Physical systems}

Students move on to a discussion of physical systems, including a ball tossed into the air and a glider oscillating on an airtrack when attached by springs to supports at the edge of the airtrack.

For the ball toss, students must predict if a ball thrown in the air is more likely to be observed at the top, middle, or bottom third of its trajectory (labeled A, B, and C; see Fig. 2). Students must interpret video of physical situations, counting the number of frames an object spends in a given

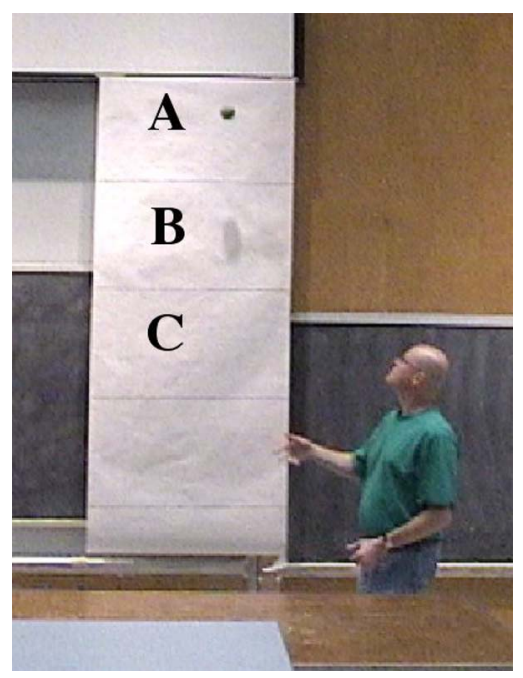

FIG. 2. (Color) Ball toss problem for discussing the probability of finding a ball in a given region when thrown vertically. 


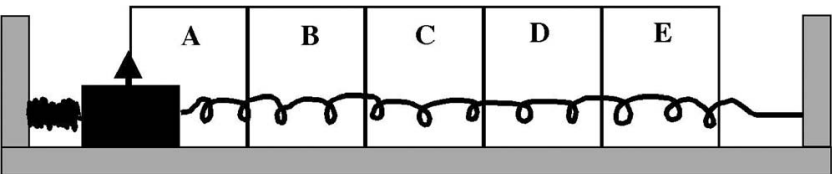

FIG. 3. Harmonic oscillator system. An air glider is attached by springs. The region of oscillation is split into five bins.

region of space. In the process, we have them extend a previous representation ${ }^{4,7}$ in which strobe photographs were considered. They bridge to the idea of probability density as the proportion of the total time spent in a region of space by comparing the number of video frames spent in a region to the total number of frames. Then, to ask for the probability of finding an object in one region of space over another, one need only consider the ratio and compare. Students qualitatively compare the speed of the ball to the probability of finding it in a region of space and find that it is most likely to be where it is moving slowest. Students often expect it to be at the bottom ("where it lands") or in the middle ("because it goes through it twice"), so the result is surprising to many.

Students end the lab-tutorial with a discussion of a harmonic oscillator. Using an air glider which is attached by springs to supports, we have created a very-low-friction situation in which students can study probability. The mathematical description given by Bao and Redish ${ }^{4}$ is far beyond the reasoning of the students in this course. Also, rather than the random-picture method, we have students simply count frames. Again, we "bin" the physical system into regions (see Fig. 3). Students use results from the ball toss problem to predict the probability of being found in a given location.

Students are asked to respond to a fictitious student dialogue in which common misunderstandings about probability $^{13,14}$ are used as some elements of reasoning while correct ideas are scattered throughout the dialogue. Incorrect ideas include the statement that region $\mathrm{C}$ is passed through more often and is therefore more likely, that region $\mathrm{C}$ is where the cart "wants to end up" and is therefore more likely, and the idea that each region is equally likely because it is equally wide.

Once students have predicted what they will observe, they count frames in a videotape of the situation. ${ }^{15}$ They use a probability histogram to plot their results. They also must compare their results to their interpretation of the student dialogue.

In lab-tutorial 7 (Probability and Energy), students revisit the ideas from lab-tutorial 5 (Probability) but interpret them more formally. First, they have developed the idea of the harmonic oscillator potential energy graph, $E_{\mathrm{pot}}=\frac{1}{2} k x^{2}$. Second, they define the concept of probability density more formally. Students are given the histogram which they found in lab-tutorial 5 and asked to discuss the relationship between potential energy, kinetic energy, and probability density.

Because the concepts of probability and probability density are revisited, we have a rich environment for studying the development of their learning. Pre-tests for lab-tutorial 7 serve as post-tests for lab-tutorial 5, for example. Data from investigations into student reasoning are given below. They are meant to illustrate the issues when teaching probability

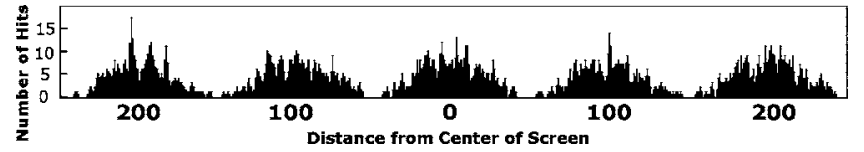

FIG. 4. Histogram of electron frequency on a screen after a simulated two-slit interference experiment. Note similarity to histogram representation in Fig. 1.

and not to make claims about effectiveness of instruction or consistency of responses.

\section{Extending probability histograms to new situations}

At the end of the lab-tutorial 7 (Probability and Energy), students participate in a full-class discussion in which they are asked to compare probability histograms from the coin toss, dice rolls, ball toss, and oscillating cart to other situations. These include the probability histogram for a frictionless cart bouncing between two perfectly elastic walls (i.e., no energy is lost in the system) and the probability histogram for electrons in a low-intensity two-slit experiment (found in a previous week's activity; see Fig. 4). This same histogram was used at the beginning of lab-tutorial 5 (Probability) to motivate a discussion of probability in the week after students first studied wave-particle duality.

\section{STUDENT UNDERSTANDING OF PROBABILITY}

Having described the teaching sequence used to help students develop an idea of probability density, we now describe students' ideas as they enter the course and how these ideas develop during instruction. We use evidence from Fall, 2005 , since students in that semester were most extensively studied. The point of our discussion is not to showcase a successful curriculum, though student understanding does improve dramatically, but to illustrate issues in teaching probability density to this specific student population.

\section{A. Understanding ensembles of easily understood events}

Before instruction on probability, we asked students a pretest in which all knew that the likelihood of a coin toss was $50-50$, heads or tails. We then asked students a multiple choice question (shown in Fig. 5) in which we asked students to explain their reasoning.

Our reason for asking the question with ten previous tosses was to help students work out the ratio of $70 \%$ heads

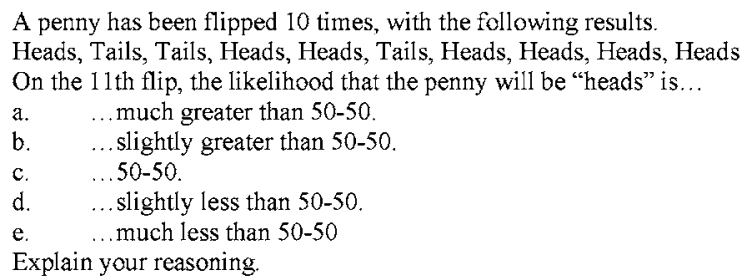

FIG. 5. Probability question. The question was designed to elicit the gambler's fallacy and other issues related to expectation values. 
TABLE II. Student responses and typical explanations on the 11th coin toss problem. $N=42$. One student gave two different explanations, so totals do not add to $100 \%$.

\begin{tabular}{lccc}
\hline \hline $57 \%$ & 24\% & $14 \%$ & $7 \%$ \\
\hline $\begin{array}{l}\text { Expect 50-50 no matter } \\
\text { what came before. }\end{array}$ & $\begin{array}{c}\text { Getting too many of a } \\
\text { kind in a row is } \\
\text { unlikely: 4 heads in a } \\
\text { row seems unlikely, so } \\
\text { tails must come soon. }\end{array}$ & $\begin{array}{c}\text { Results should end up } \\
\text { at 50-50, so tails is } \\
\text { likely in the future. }\end{array}$ & $\begin{array}{c}\text { Past distribution guides } \\
\text { future: since past } \\
\text { results are 70-30 H-T, } \\
\text { we should continue to } \\
\text { have that ratio in the } \\
\text { future. }\end{array}$ \\
\hline \hline
\end{tabular}

and $30 \%$ tails. We used a $70-30$ ratio because $60-40$ is too close to 50-50 and perhaps not sufficiently different from the expected 50-50. We wished the result of the first ten tosses to be unexpected but not outrageously so (as ten consecutive tosses of heads would have been). A correct answer is (c), 50-50, with the reasoning that previous tosses of the coin have no effect on an event. Thus, for a fair coin, one can both ignore the ensemble of past results and also not compare the ensemble to the eventual, expected result. A majority of students gave the correct answer, but many gave other responses (see Table II). The results show that many students enter our courses with everyday expectations about what the ensemble values of sets of coin tosses should be and use past results to decide future events. Our results are consistent with those found by Bao when discussing similar problems. ${ }^{16}$

\section{B. Interpretations of probability density for physical systems}

We asked a series of questions (often identical) throughout the semester to investigate the development of student reasoning about probability density in physics systems. We note that pretests and post-tests were never handed back to students, and only the tutorials and tutorial homework were in students' hands as they studied for examinations.

\section{In a gravitational setting}

Before any instruction on probability, students were asked the question shown in Fig. 6 on an ungraded lab-tutorial pretest. The goal of the question was to find what methods students used for determining the likelihood of finding an object in space. Students are told that the falling raindrop is constantly speeding up, so it is most likely to be in region A where it spends the most time.

We discuss three answers from this pretest. One-third of the 42 students answering the question gave the right answer with the correct reasoning. We group other responses into two major categories.

Random observation of equal sized bins means equal likelihood. Slightly more than 1/3 (15 of 42) of the students said that the drop was equally likely to be found in each region. The reasoning given was either that equal-sized bins lead to equal probabilities or that the random nature of the observation made each region equally likely. Neither explanation
An extremely light rain is falling out of a SmartCloud, such that only one drop of rain is in the air at a time, and as soon as one drop hits the ground, another is released from the cloud. (See the picture below.) Each drop speeds up continually until it hits the ground. The space between the cloud and the ground is divided into three equal-size regions, A, B, and C. 100 pictures are taken of this system at random times. The majority of the photographs will show the raindrop in...

\section{a. Region $\mathrm{A}$}

b. Region B

c. Region $\mathrm{C}$

d. There will be about an equal number of each.

Explain your reasoning$$
\text { Drop starts falling. }
$$

A little while later...

And a little later...

Drop reaches the ground and another starts...

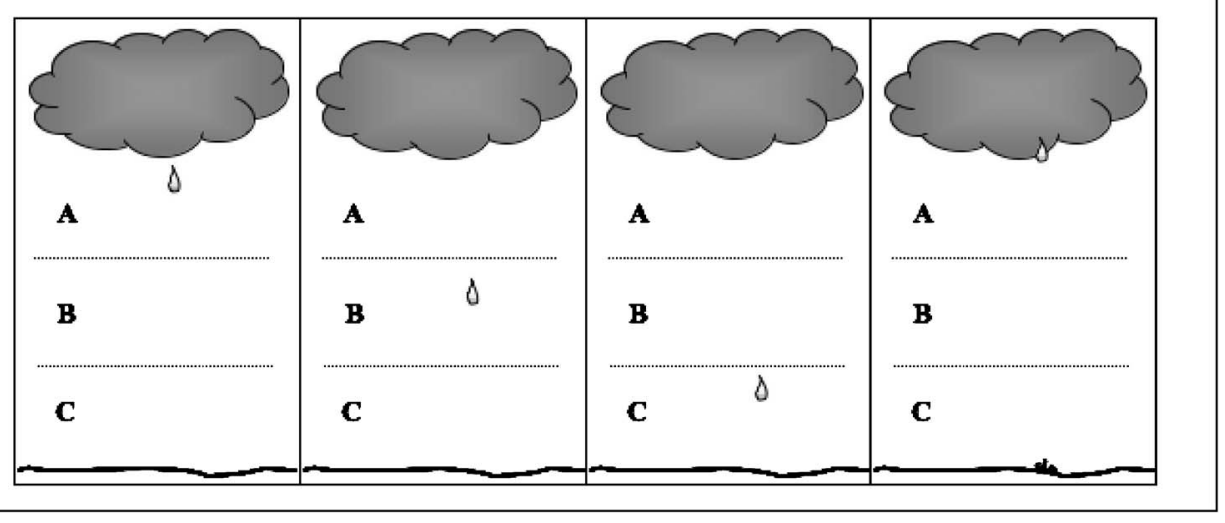

FIG. 6. Pre-test question on probability density. Students were asked to predict where a single falling raindrop (constantly speeding up) was most likely to be observed. 


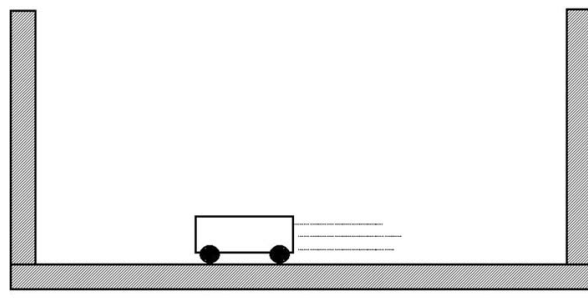

Imagine a situation where a small cart is rolling back and forth between two walls Assume the cart rolls at constant speed. When it hits a wall, it turns around and moves in the opposite direction at the same speed with which it hit the wall.

\section{Sketch the probability density of the} cart.

FIG. 7. "Free cart" probability density question. Students answered this (or a very similar) question on a pre-test, post-test immediately after instruction, and examination.

was found again after instruction, though the former may have been helpful when discussing the "free cart," described below.

Starting or ending issues. Roughly $1 / 5$ (8 of 42) of the students described the most likely scenario as either region $\mathrm{A}$ or $\mathrm{C}$ because the drop was starting or ending there, respectively. We believe that these students are not looking at the entire motion of the drop and therefore cannot make the comparison of "time in a region" to "total time for drop to fall." Thus, they use other reasoning, in this case the idea that starting or ending points are more likely to be observed. We note that another two students $(5 \%)$ described B as the most likely area because it is in the middle. This response was given more commonly in classroom situations in lab-tutorial 5, when a ball tossed up and down was considered; students described the ball passing through region $\mathrm{B}$ twice, for example. Results are similar to those by Ambrose with more advanced students. ${ }^{13,14}$

\section{2. "Free cart" between hard walls}

We refer to a cart bouncing back and forth between two hard walls and never losing energy as the "free cart," in comparison to a cart attached to springs (the "harmonic cart") or a cart on a ramp. Students studied the harmonic cart in lab-tutorial, and we chose to ask questions about a simpler scenario when pre- and post-testing them. The basic question on the three tests was essentially identical (see Fig. 7), though the post-test and examination contained additional elements which are mentioned below, as needed.

The pre-test was given the week before lab-tutorial 7 (Probability and Energy). As noted above, it served in part as a post-test of student learning in lab-tutorial 5 (Probability). The post-test was given as an ungraded quiz on the first day of lecture after all students had completed lab-tutorial 7. The examination was given six weeks later, near the end of the semester. During those weeks, students had worked primarily on the interpretation of probability density in the context of
TABLE III. Student responses on the free cart problem.

\begin{tabular}{lccc}
\hline \hline & $\begin{array}{c}\text { Pre-test } \\
N=38\end{array}$ & $\begin{array}{c}\text { Post-test } \\
N=41\end{array}$ & $\begin{array}{c}\text { Examination } \\
N=43\end{array}$ \\
\hline Equal in all & $52 \%$ & $52 \%$ & $88 \%$ \\
Higher at edges & $33 \%$ & $48 \%$ & $10 \%$ \\
\hline \hline
\end{tabular}

the Schrödinger equation. There was lecture discussion on the differences in probability density between an electron in the ground state of a finite square well and the free cart between hard walls, so the topic was revisited in lecture after lab-tutorial instruction.

Data from the three tests are shown in Table III. Note that we include only the two most common responses and do not include the rare other responses in the case of the ungraded post-test. Students giving the correct response (equally likely in all regions of space) also gave correct reasoning by saying that the speed is the same in all regions and the time spent in all regions is equal. We describe four specific results from this table.

Hidden variables in the prevalence of correct answers. We do not have sufficient data to understand either the lack of change from pre- to post-test nor the increase in results from the post-test to the examination and believe several variables may play a role. The post-test was administered the day after laboratory; students had not yet completed the homework and had not had time to practice the ideas. On the homework, students had to sketch the probability density for a runner moving at different speeds. Furthermore, the tutorials on tunneling included a discussion of the "magnetic cart," in which a cart with magnets attached passes through a potential barrier made by a strong magnetic field. In addition, instruction during lecture time emphasized comparisons between the quantum particle in a finite square well and the "free cart." Finally, it is possible that the difference in students attitudes toward an ungraded post-test and a graded examination question also played a role. Each of these may have had an effect in increasing student understanding of probability density.

Three different issues arise when analyzing the increase in the incorrect response in the post-test.

Pattern matching. From the student responses, it seems that many on the pre- and post-test were pattern matching to the harmonic oscillator which had already been discussed in lab-tutorial 5 and was again discussed in lab-tutorial 7. We note that our analysis does not include those students who correctly discussed carts slowing down to turn around; their graphs were notably different from those who pattern matched to the harmonic oscillator. The responses we counted as "higher at edges" were mostly parabolas or similar shapes, some actually identical to the histograms drawn in lab-tutorial. We believe students are pattern matching to previously taught material without considering the actual situation. That more pattern match after lab-tutorial 7 than before is worrisome, but consistent with the expectations that many students have ${ }^{10}$ that one learns physics by memorizing and not by thinking through a situation.

Counting issues and edge effects. Many students in a 
Consider the experiment shown below. A series of balls is set moving towards the right at a very small velocity $y_{0}$. (Ignore friction.) On level 2 , a ball moves twice as fast as on level 1 .

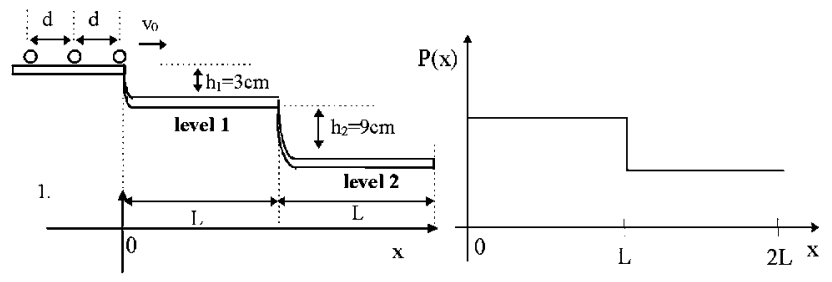

1. On the axes above, sketch the probability density of finding a ball.

2. Explain how you arrived at the shape that you have drawn in question 1 .

FIG. 8. "Ball on ramps" probability density question. Students answered this question on the final examination. A correct graph is shown on the right.

classroom discussion following the post-test supported the idea that the free cart was more likely to be found at the edges because it was in that region of space both on its way to the wall and again after bouncing off. It was there twice for every one time in another region. Other students during the class discussion made the correct point that one needed to compare "time in a region" to the accurate "total time"namely, for a full cycle of the cart back and forth in both directions. Thus, the cart was in each region twice, not just those regions at the edges. That the cart was in regions at the edges twice in a row did not matter. This point was accepted in the large-lecture discussion, but may have been caused by activities in the lab-tutorial itself. When counting frames in a video, one counts all frames for a region at the edge consecutively, and might treat the regions away from the edges differently.

Limited types of responses. Finally, we note that no other responses than those shown in the table "survived" the instructional process. Though the pre-tests contained some other (not shown) responses, these were no longer given after instruction. The lasting issue for students remains one of understanding the physical situation. Either students patternmatched to the wrong system or they had problems understanding which region of space to consider. It is also possible that students switched from one wrong answer to another. We note that 8 of the 22 students who gave the correct answer on the pre-test switched to the incorrect answer of "higher at the edges" on the post-test. With two, it is clear they were pattern matching to the harmonic oscillator situation, while the sketches and explanations of the other six do not allow us to distinguish what guided their reasoning.

\section{Ball on stepped ramps}

On the final examination, students answered a question taken from Bao and Redish ${ }^{4}$ (see Fig. 8). In the question, students must analyze the speed of the object on different levels, recognize that the levels are of equal length, and compare the time on each level to the total time for a ball to traverse the path. A correct answer would show $P(x)$ twice as high from 0 to $L$ as from $L$ to $2 L$.

We counted qualitatively correct responses (without the explicit 2-to-1 ratio) as correct in our analysis, as long as the
TABLE IV. Student responses on the final examination balls-onramps question, $N=44$.

\begin{tabular}{lc}
\hline \hline & Final exam \\
\hline Correct with 2:1 ratio & $45 \%$ \\
Qualitatively correct & $38 \%$ \\
Sloped line in one region & $14 \%$ \\
\hline \hline
\end{tabular}

students' explanations qualitatively correctly described relative speeds and times on each level, but list them separately in Table IV. We did not accept student responses in which they seemed to map the physical picture to the ramps to the $P(x)$ graph. Of the 44 students answering the question, 83\% were completely or qualitatively correct with correct reasoning, while $14 \%$ showed some sort of sloped line, usually on level 2. Other responses (not listed in the table) were given by only one or two students at a time.

\section{DISCUSSION OF RESEARCH AND INSTRUCTION}

When describing the likelihood of finding a particle in one region of space over another, students must reason about time in a region compared to the total time for an event to occur, meaning they must understand the physical system well enough to compare speeds in regions of space and to understand how long the event takes. Our teaching materials are designed to help students develop these ideas. But students often use other methods to guide their reasoning, even after instruction. They may pattern match to different systems that have been studied in detail. They may focus on consecutive events, such as carts turning around when bouncing off walls. They may also look at specific times during the event, such as its beginning or its end. Finally, students may use correct reasoning but not be able to accurately calculate the times involved. In each case, we can see what they fail to do, but we emphasize what they actually do as a way of better understanding what valuable ideas they have that might be built on in future modifications to our instruction.

We find that there is improvement in student reasoning about probability density, but that teaching only about the classical systems (including graphs of their potential energies) was not sufficient to help students learn the material. Post-test results may have too quickly followed instruction and show that students did not immediately change their ideas about probability density in a very simple system, the free cart. Later results, on an examination near the end of the semester and a final examination, show that students developed a more complete understanding and that more than $80 \%$ were able to answer questions about probability density after additional instruction in simple systems in quantum physics. We note that 29 of the 37 students who correctly answered the examination question about the free cart also answered the balls on ramps question correctly (meaning that nearly $80 \%$ were consistent in their correct answers). We believe that having $80 \%$ of the class, on a given question, discuss a 
difficult concept accurately, and to have $80 \%$ of that population do so consistently, is an indicator of a successful class, especially when only $1 / 3$ of the students could accurately describe the situation before instruction.

\section{ACKNOWLEDGMENTS}

Katrina E. Black, David E. Clark, and Eleanor C. Sayre contributed to the development of the instructional materials used in the lab-tutorials. We used DataStudio software, from Pasco, but the experiments can easily be created using other, similar software packages. We thank Rachel E. Scherr, John R. Thompson, and R. Padraic Springuel for discussions about and contributions to the course and this paper. Development work on this course is supported in part by NSF Grant No. DUE-0410895.
*FAX: (207) 581-3410. Email address: wittmann@umit.maine.edu ${ }^{1}$ J. Garfield and A. Ahlgren, Difficulties in Learning Basic Concepts in Probability and Statistics: Implications for Research, J. Res. Math. Educ. 19, 44 (1988).

${ }^{2}$ C. Konold, Issues in Assessing Conceptual Understanding in Probability and Statistics, J. Educ. Stat. 3, http:// www.amstat.org/publications/jse/v3n1/konold.html (1995).

${ }^{3}$ H. Sahai, A. Khurshid, and S. C. Misra, A Second Bibliography of the Teaching of Probability and Statistics, J. Educ. Stat. 4, http://www.amstat.org/publications/jse/v4n3/sahai.html (1996).

${ }^{4}$ L. Bao and E. F. Redish, Understanding probabilistic interpretations of physics systems: A prerequisite to learning quantum physics, Am. J. Phys. 70, 210 (2002).

${ }^{5}$ B. S. Ambrose et al., An investigation of student understanding of single-slit diffraction and double-slit interference, Am. J. Phys. 67, 146 (1999).

${ }^{6}$ D. Domert, C. J. Linder, and Å. Ingerman, Probability as a conceptual hurdle to understanding one-dimensional quantum scattering and tunnelling, Eur. J. Phys. 26, 47 (2005).

${ }^{7}$ P. Jolly et al., Visualizing motion in potential wells, Am. J. Phys. 66, 57 (1998).
${ }^{8}$ M. C. Wittmann et al., http://www.arxiv.org/abs/physics/0602141

${ }^{9}$ R. R. Harrington and E. E. Prather, in The Changing Role Of Physics Departments In Modern University, AIP Conf. Proc. No. 399, edited by E. F. Redish and J. Rigden (AIP, Woodbury, 1997), p. 797. Available online at http://perlnet.umaine.edu/bio/ harrington/ICUPE.html

${ }^{10}$ M. C. Wittmann, Lab-Tutorials fur den Quantenphysik Unterricht, Prax. Naturwiss., Phys. Sch. 55, 16 (2006), http://arxiv.org/abs/ physics/0604115

${ }^{11}$ E. F. Redish and T. McCaskey (personal communication).

${ }^{12}$ E. F. Redish, J. M. Saul, and R. N. Steinberg, Student expectations in introductory physics, Am. J. Phys. 66, 212 (1998).

${ }^{13}$ B. S. Ambrose, Student Understanding of Probability in Classical and Modern Physics, AAPT Announcer 31 (2), 81 (2001).

${ }^{14}$ B. S. Ambrose, Student Understanding of Probability in the Classical and Semiclassical Regimes, AAPT Announcer 32 (2), 134 (2002).

${ }^{15}$ Video available at http://perlnet.umaine.edu/abt/v2video.htm

${ }^{16}$ L. Bao, Ph.D. thesis, available at http://physics.umd.edu/perg/ dissertations/, University of Maryland, 1999. 